\title{
The formation of sunspot penumbra
}

\section{Magnetic field properties}

\author{
R. Rezaei, N. Bello González, and R. Schlichenmaier
}

Kiepenheuer-Institut für Sonnenphysik, Schöneckstr. 6, 79104 Freiburg, Germany

e-mail: [rrezaei; nbello; schliche]@kis . uni-freiburg.de

Received 15 June 2011 / Accepted 7 October 2011

\begin{abstract}
Aims. We study the magnetic flux emergence and formation of a sunspot penumbra in the active region NOAA 11024.

Methods. We simultaneously observed the Stokes parameters of the photospheric iron lines at $1089.6 \mathrm{~nm}$ with the TIP and $617.3 \mathrm{~nm}$ with the GFPI spectropolarimeters along with broad-band images using G-band and Ca II K filters at the German VTT. The photospheric magnetic field vector was reconstructed from an inversion of the measured Stokes profiles. Using the AZAM code, we converted the inclination from line-of-sight (LOS) to the local reference frame (LRF).

Results. Individual filaments are resolved in maps of magnetic parameters. The formation of the penumbra is intimately related to the inclined magnetic field. No penumbra forms in areas with strong magnetic field strength and small inclination. Within $4.5 \mathrm{~h}$ observing time, the LRF magnetic flux of the penumbra increases from $9.7 \times 10^{20}$ to $18.2 \times 10^{20} \mathrm{Mx}$, while the magnetic flux of the umbra remains constant at $\sim 3.8 \times 10^{20} \mathrm{Mx}$. Magnetic flux in the immediate surroundings is incorporated into the spot, and new flux is supplied via small flux patches (SFPs), which on average have a flux of $2-3 \times 10^{18} \mathrm{Mx}$. The spot's flux increase rate of $4.2 \times 10^{16} \mathrm{Mx} \mathrm{s}^{-1}$ corresponds to the merging of one SFP per minute. We also find that, during the formation of the spot penumbra, a) the maximum magnetic field strength of the umbra does not change; b) the magnetic neutral line keeps the same position relative to the umbra; c) the new flux arrives on the emergence side of the spot while the penumbra forms on the opposite side; d) the average LRF inclination of the light bridges decreases from $50^{\circ}$ to $37^{\circ}$; and e) as the penumbra develops, the mean magnetic field strength at the spot border decreases from 1.0 to $0.8 \mathrm{kG}$.

Conclusions. The SFPs associated with elongated granules are the building blocks of structure formation in active regions. During the sunspot formation, their contribution is comparable to the coalescence of pores. Besides a set of critical parameters for the magnetic field, a quiet environment in the surroundings is important for penumbral formation. As remnants of trapped granulation between merging pores, the light bridges are found to play a crucial role in the formation process. They seem to channel the magnetic flux through the spot during its formation. Light bridges are also the locations where the first penumbral filaments form.
\end{abstract}

Key words. magnetic fields - techniques: polarimetric - Sun: photosphere - Sun: surface magnetism - sunspots - Sun: evolution

\section{Introduction}

The Sun is a unique laboratory for studying cosmological magnetic fields. Active regions (AR) are manifestations of large-scale magnetic field in the solar atmosphere. The largest magnetic structures in ARs are sunspots. To understand the formation of such magnetic features, one has to study the fundamental process of flux emergence (Lites 2009; Kosovichev 2009). The rise of buoyant magnetic flux tubes is the basic picture of the present understanding of these processes. Since its introduction by Parker (1955), there have been many attempts to simulate the emergence of buoyant flux tubes in the convection zone (Caligari et al. 1995; Abbett et al. 2000; Fang et al. 2010). As a result of flux emergence, pores and other smaller magnetic structures also form in ARs. An understanding of their structure and evolution is a necessity for solving the problem of magnetic field generation in the solar interior (Parker 1979). The formation of the sunspot penumbra and the associated onset of the Evershed flow (Leka \& Skumanich 1998; Yang et al. 2003 ) is of particular interest in this respect as summarized in the reviews by Solanki (2003), Weiss (2006), and Schlichenmaier (2009). Recently, numerical simulations try to reproduce the flux-emergence phenomena in the solar atmosphere (Cheung et al. 2008; Martínez-Sykora et al. 2009; Tortosa-Andreu \& Moreno-Insertis 2009; Cheung et al. 2010; Fang et al. 2010).

We succeeded in acquiring a spectropolarimetric data set of a forming penumbra (NOAA 11024) with high temporal cadence and spatial resolution (Schlichenmaier et al. 2010a,b, hereafter Papers 1 and 2). The image sequences in the G-band and in the Ca II K filters show that the penumbra forms in segments at the outer boundary of the protospot on the side away from to the AR center. As the area of the penumbra increases, the area of the umbra stays approximately constant (Paper 1). Although Zwaan (1985, 1987) compiled observations suggesting that sunspots form out of merging pores, we concluded in Paper 2 that a fraction of the magnetic flux required to form a sunspot emerges in the form of granular-scale bipoles between the two polarities of the AR. These bipoles are cospatial with elongated granules as seen in intensity. Subsequently the bipole extremes separate and the proper polarity merges with the forming spot, therefore the magnetic flux of the spot increases and the penumbra forms.

In this Paper, we quantify the magnetic properties of the elongated granules and the spot as it develops a penumbra. The Evershed flow and velocities will be addressed in the next 
paper. We present a time series of spectropolarimetric maps that were taken simultaneously with the imaging data presented in Paper 1. The data sets of our two spectropolarimeters the (visible) GREGOR Fabry-Pérot 2D-interferometer (GFPI) and the Tenerife Infrared Polarimeter(TIP II) are described in Sect. 2. The Stokes profiles are inverted to retrieve the physical parameters (cf. Sect. 3.2). A comparison between maps of the two independent spectropolarimeters highlights the reliability of our measurements. In Sect. 4 we elaborate on the increase in magnetic flux as the protospot transforms into a sunspot. Section 5 summarizes and discusses our analysis, while Sect. 6 presents the conclusions.

\section{Observations}

As discussed in Papers 1 and 2, the evolution of the AR 11024 was observed on consecutive days in July 2009 at the German Vacuum Tower Telescope (VTT, Schröter et al. 1985). From SoHO/MDI images (Scherrer et al. 1995), it is seen that two pores emerged on July 3 and formed a protospot (Schlichenmaier et al. 2011). In this contribution, we focus on the July 4 observations, during which the leading spot of the AR, located at a heliocentric angle $\theta \sim 28^{\circ}$, developed a penumbra.

This observing campaign was based on a multi-instrument, multiwavelength optical setup like the ones reported by Beck et al. (2007) for TIP/TESOS (see also Kucera et al. 2008). However, this was the first simultaneous TIP/GFPI observing campaign. We combined the TIP II (Collados et al. 2007) attached to the Echelle spectrograph, the GFPI (Puschmann et al. 2006; Bello González \& Kneer 2008), and two speckle-imaging channels, in the G-band and Ca II K. A dichroic beam-splitter was used to feed GFPI with visible light and TIP with infrared. A small fraction of the visible beam was extracted for both imaging systems. The scanning mirror of the Kiepenheuer Adaptive Optics System (KAOS, von der Lühe et al. 2003) was used for spatial scanning by shifting the solar image at the entrance of the spectrograph slit. Thus, one could built maps of a certain area of the Sun with the slit spectrograph while scanning in wavelength with the 2D spectrometer and imaging in the speckle channels. The filtergrams in the G-band and $\mathrm{Ca}$ II $\mathrm{K}$ were reconstructed using the KISIP code (Wöger \& von der Lühe 2008; Wöger et al. 2008), achieving a spatial resolution better than 0.'3. In Paper 1 , properties of the sunspot formation as seen in the reconstructed image sequences have been presented. Two movies are available as online material in Paper 1.

\subsection{One-dimensional spectropolarimetric data}

TIP recorded maps in the Fe I line at $1089.6 \mathrm{~nm}$ (Table 1). The line forms only in a small height range, such that an analysis that assumes that magnetic field and velocity are constant across the formation range is more reliable than, e.g., for Si I $1082.7 \mathrm{~nm}$ which has contributions from the entire photosphere. Two full sunspot maps were recorded between 10:42-10:58 and 11:43-11:59UT with a slit width corresponding to 0.3 and a step size of 0.3 , a scanning range of $24^{\prime \prime}$ (i.e., 80 steps) and a slit length of $78^{\prime \prime}$. During the rest of the observing time, we performed scans with a range of only 2 '. 1 to keep the spot within the field of view (FOV) of the GFPI, which recorded data continuously. The spatial sampling along the slit after binning amounts to 0.35 . The spectral sampling is $1.1 \mathrm{pm}$, and the effective exposure time per slit position was $10 \mathrm{~s}$. The polarimetric calibration was performed using the VTT telescope model and the nearinfrared instrumental calibration unit (Beck et al. 2005a). The
Table 1. Atomic properties of the observed spectral lines (Nave et al. 1994).

\begin{tabular}{lcccc}
\hline \hline Line & $\lambda(\mathrm{nm})$ & Exc pot $(\mathrm{eV})$ & $\log (g f)$ & $g$-effective \\
\hline $\mathrm{Fe}_{\mathrm{I}}$ & 1089.63 & 3.071 & -2.85 & 1.50 \\
$\mathrm{Fe}_{\mathrm{I}}$ & 617.334 & 2.223 & -2.88 & 2.50 \\
\hline
\end{tabular}

residual crosstalk was corrected for using the statistical method that is described in Schlichenmaier \& Collados (2002). The $1 \sigma$ noise level in the TIP data is about $1 \times 10^{-3} I_{\mathrm{c}}$.

\subsection{Two-dimensional spectropolarimetric data}

The GFPI system scanned 31 spectral points along the Fe I line at $617.3 \mathrm{~nm}$ (Table 1) with a wavelength sampling of $1.48 \mathrm{pm}$, recording the four Stokes parameters. The observations were taken in speckle mode, i.e. seven frames of short exposure $(20 \mathrm{~ms})$ per spectral position and polarimetric state, simultaneously taken with broad-band images. Using this method, we achieved a $1 \sigma$ noise level of about $8 \times 10^{-3} I_{c}$. The data have been filtered in wavelength to reduce noise and the residuals of the fluctuations of the spectrometer transmission by applying a filter with a FWHM of $4.9 \mathrm{~nm}$ in the Fourier domain (see Bello González et al. 2009, Appendix A). An upgraded version of the "Göttingen" speckle code originally developed by de Boer (1996) was applied to restore the broad-band data. This code takes into account the variations in the adaptive optics correction from the lock point in a similar way as explained in Puschmann $\&$ Sailer (2006). The spectropolarimetric data were then restored following Keller \& von der Lühe (1992), achieving a spatial resolution of better than 0.' 4 when seeing allowed. To minimize the cadence to $56 \mathrm{~s}$, the readout time was reduced by exposing only half of the FOV of the GFPI detector.

We took care of the image shifts due to simultaneous scanning steps of TIP by aligning the individual images. The reduced FOV typically amounts to $33^{\prime \prime} \times 22^{\prime \prime}$ with a spatial sampling of 0 .' 109 per pixel. The output of the GFPI narrow-band channel is a data cube containing spatial and spectropolarimetric information as a linear combination of four polarimetric states (Bello González \& Kneer 2008). To retrieve the Stokes parameters, the data are demodulated by applying the inverse Mueller matrix of the telescope-KAOS-GFPI optical train. The telescope Mueller matrix is calculated following the VTT model by Beck et al. (2005a). The Mueller matrix of the KAOS system plus the GFPI modulator (polarimeter) is obtained from the calibration performed with the visible instrumental calibration unit as described in Beck et al. (2005b). The estimated efficiencies on 2009 July 4 at $\sim 617.3 \mathrm{~nm}$ are, for both orthogonal ${ }^{1}$ polarimetric states, $\epsilon_{1}=(0.38,0.43,0.57)$ and $\epsilon_{2}=(0.45,0.44,0.59)$, respectively. The data were corrected for crosstalk in the same manner as the TIP data.

\subsection{Line properties}

The Fe I line at $1089.6 \mathrm{~nm}$ has a line-core intensity of about $78 \% I_{c}$ (Kurucz, et al. 1984). We measure a line-core intensity of $83 \% I_{\mathrm{c}}$ at a heliocentric angle of $28^{\circ}$ with TIP $(82 \%$ at disk center). The small discrepancy can be explained by the presence

1 The GFPI polarimeter contains a polarizer beam-splitter that divides the incoming light into the ordinary and extraordinary (orthogonal) beams. The advantages of this system are discussed in e.g., Bello González \& Kneer (2008). 


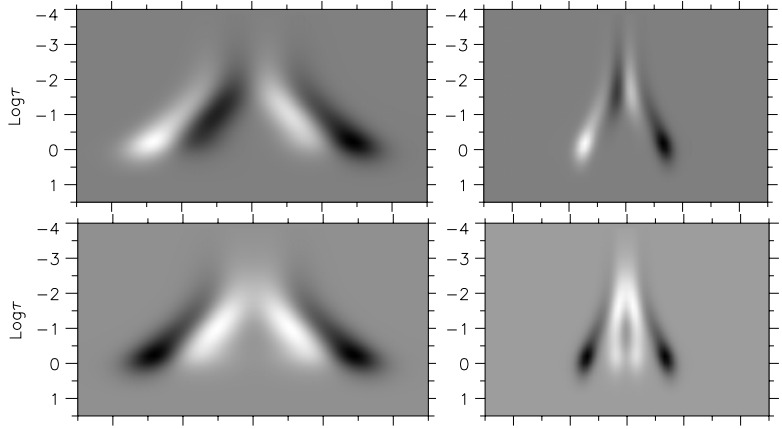

Fig. 1. Response of $V(\lambda)$ to LOS velocity (upper row) and magnetic field strength (lower row) for Fe $1089.6 \mathrm{~nm}$ (left column) and Fe I $617.3 \mathrm{~nm}$ (right column). Each tick mark in the $x$-axis corresponds to $10 \mathrm{pm}$.

of parasitic light (non-dispersed stray light) in the Echelle spectrograph (5\% in the visible, Beck \& Rezaei 2011) as well as by the existence of (dispersed) stray light (10\%, Beck 2008).

The line depression response function for Stokes $I$ at line minimum peaks at $\log \tau=-1(150 \mathrm{~km})$ and the triplet Landéfactor amounts to 1.5 , making $\mathrm{Fe}_{\mathrm{I}} 1089.6 \mathrm{~nm}$ a good choice to investigate the magnetic and velocity patterns in the lower photosphere (see Table 1). The Fe I line at $617.3 \mathrm{~nm}$ has a line minimum of $36 \% I_{c}$ (cf. BASS2000, Paletou et al. 2007), and the GFPI measures a line minimum of $43 \% I_{\mathrm{c}}$ at disk center. The response function at line minimum peaks at $\log \tau=-1.5(230 \mathrm{~km})$, and the triplet Landé-factor amounts to 2.5. Therefore this line is well suited to studying magnetic fields at midphotosphere. Figure 1 displays the response functions for the Stokes- $V$ profiles of the two lines (cf. Cabrera Solana et al. 2005). The values of the $2 \mathrm{D}$ response function is decoded in gray scale and displayed in $\log \tau$ versus $\Delta \lambda$. The response functions are calculated for a penumbra atmosphere with the magnetic and thermal stratifications from del Toro Iniesta et al. (1994) and Bellot Rubio et al. (2006), respectively. While Fe I $1089.6 \mathrm{~nm}$ shows a sizable response for $\log \tau>-2$, Fe I $617.3 \mathrm{~nm}$ responds to changes in a slightly broader depth range $\log \tau>-2.5$.

\subsection{Time coverage}

The sunspot evolution on July 4 was followed from 08:09 to 12:55 UT with the GFPI, 07:58 to $13: 11$ with TIP, and 08:32 to 13:07 with the two imaging cameras.

\section{Data analysis}

\subsection{Typical data set}

A snapshot of the NOAA 11024 leading spot as seen in Stokes $(I, Q, U, V)$ at a late stage is shown in Fig. 2. The data were taken quasi-simultaneously with GFPI (upper row) and TIP (lower row). The TIP scanning direction is from the bottom to the top of the map. The contours that outline the spot were constructed manually from intensity maps. Although the spatial resolution of the TIP maps is lower than that of GFPI maps, and although the lines have a slightly different formation height, the maps compare very well. This comparison reassures us that the spectropolarimetric capabilities of the GFPI are suited to retrieve the physical parameters of the solar atmosphere. Yet, one should keep in mind that the noise level in TIP data is lower by a factor of 8 .

\subsection{Inversion of Stokes profiles}

An inversion was performed using the SIR code (Ruiz Cobo \& del Toro Iniesta 1992; Bellot Rubio 2003) for TIP and GFPI data, separately. Some authors use two magnetic components in the inversion of sunspot penumbra (e.g., Bellot Rubio et al. 2004; Beck 2011). In order to keep the number of free parameters as small as possible, we did not use such a two-component setup. Instead, we used one magnetic component plus stray light, for the data of both instruments as in e.g., Puschmann et al. (2010). Such a one component and height-independent inversion setup has a slight tendency to underestimate the magnetic flux values in locations where strong gradients or opposite polarities (Rezaei 2008; Franz 2011) are present (Bellot Rubio et al. 2003).

The fraction of stray light was set to $15 \%$ for TIP (Beck 2006, Sect. 5.4) and 12\% for GFPI (Bello González 2006, Sect. 3.2), respectively, of an average quiet-Sun profile. Cabrera Solana et al. (2007) and Rezaei et al. (2007) report similar values, but using different methods and definitions (see also, Allende Prieto et al. 2004; Beck et al. 2011). In the single magnetic component, the magnetic field, the velocity, and the values for macro- and micro-turbulence were constant with height. Hence our inversion aims to estimate the average values of magnetic and velocity fields. We do not aim at modeling the asymmetries of the line profiles being produced by gradients along the LOS or by unresolved components.

Profiles of similar penumbral locations are compared in Fig. 3. It demonstrate that the Fabry-Pérot spectropolarimeter is capable of recording complex profiles with only 31 wavelength points. As seen in Fig. 3, the symmetric parts of the observed lines are well fitted, and we retrieve reliable averages of the velocity and magnetic field parameters over the formation height of the lines.

Maps of the magnetic field strength, LOS inclination, magnetic flux along the LOS, and LOS velocity (as retrieved from the data sets of Fig. 2) are compared in Fig. 4. The upper row corresponds to GFPI and the lower one to TIP. The color codings for the maps of each quantity are identical for the two instruments except for the magnetic flux, which is the flux per pixel. Since the pixels of TIP correspond to an area on the solar surface, which is about a factor of 9 larger, the color coded values for TIP are rescaled correspondingly.

\subsection{Transformation to local reference frame (LRF)}

We convert the magnetic field inclination and azimuth from the LOS frame to the LRF using the known values of the position of the spot on the solar disk. Due to the intrinsic $180^{\circ}$ ambiguity in azimuth (Metcalf 1994; Lites et al. 1995), we have two sets of solutions. Using the AZAM program (Elmore et al. 1992; Tomczyk et al. 1992; Metcalf et al. 2006), we removed the ambiguity and reached the final results.

\section{Magnetic evolution}

A sequence of six snapshots tracking the formation of the penumbra is shown in Fig. 5. The contours were drawn manually as the intensity boundary of the spot. From left to right we display maps of intensity, field strength $(\mathrm{kG})$, magnetic flux (LOS, $10^{17} \mathrm{Mx}$ ), and inclination (LOS, degrees). From top to bottom time evolves from 08:40, 08:50, 09:28, 10:13, 11:51, and 12:58 UT. A legend for the color coding is displayed at the top of each column. An additional set of maps at 11:54 UT is shown in the top row of Fig. 4. 

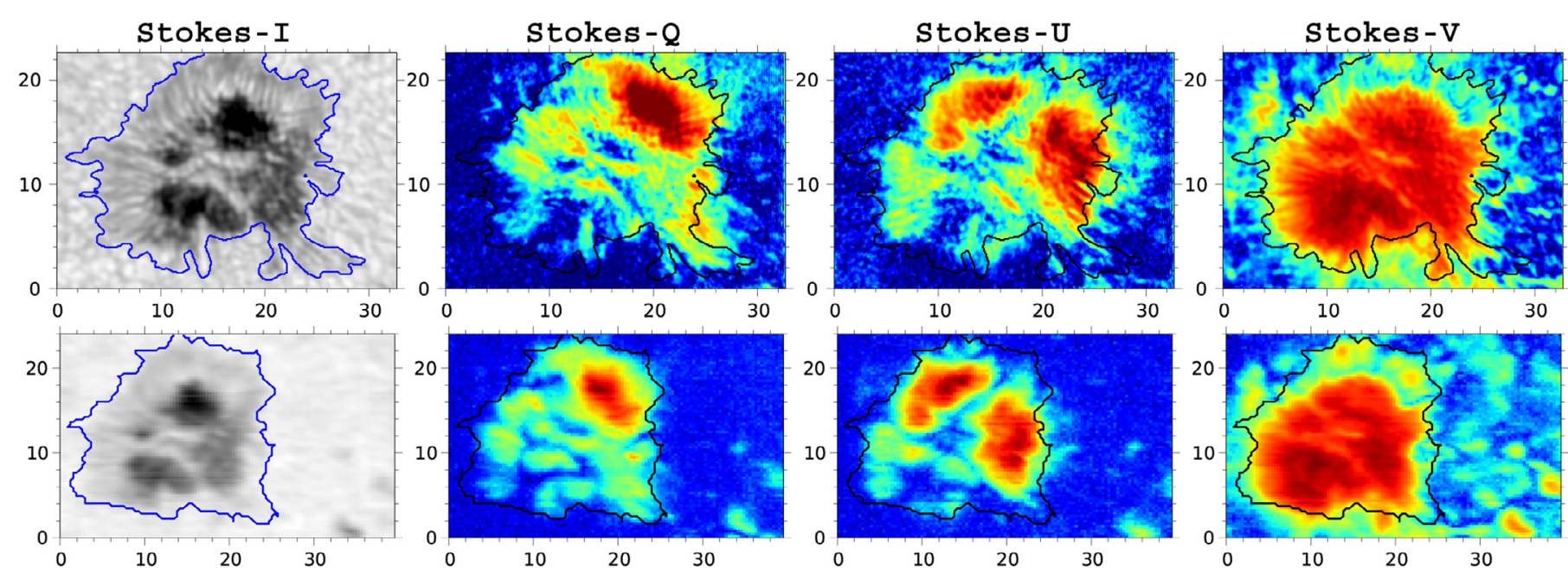

Fig. 2. Quasi-simultaneous polarimetric maps of GFPI (Fe I $617.3 \mathrm{~nm}$, top) and $\mathrm{TIP}\left(\mathrm{Fe}_{\mathrm{I}} 1089.6 \mathrm{~nm}\right.$, bottom). From left to right: maps of continuum intensity, $I\left(\lambda_{\mathrm{c}}\right)$, and the polarimetric states $\int|X(\lambda)| \mathrm{d} \lambda, X \in\{Q, U, V\}$. Contours show the corresponding continuum boundary of the spot (manually drawn). The TIP maps should to be rotated by $3^{\circ}$ in clockwise direction to be aligned with GFPI maps. The GFPI data was acquired at 11:54, while the TIP map was scanned from 11:43 until 11:59 UT (from bottom to top). Tick marks are in arcsec.
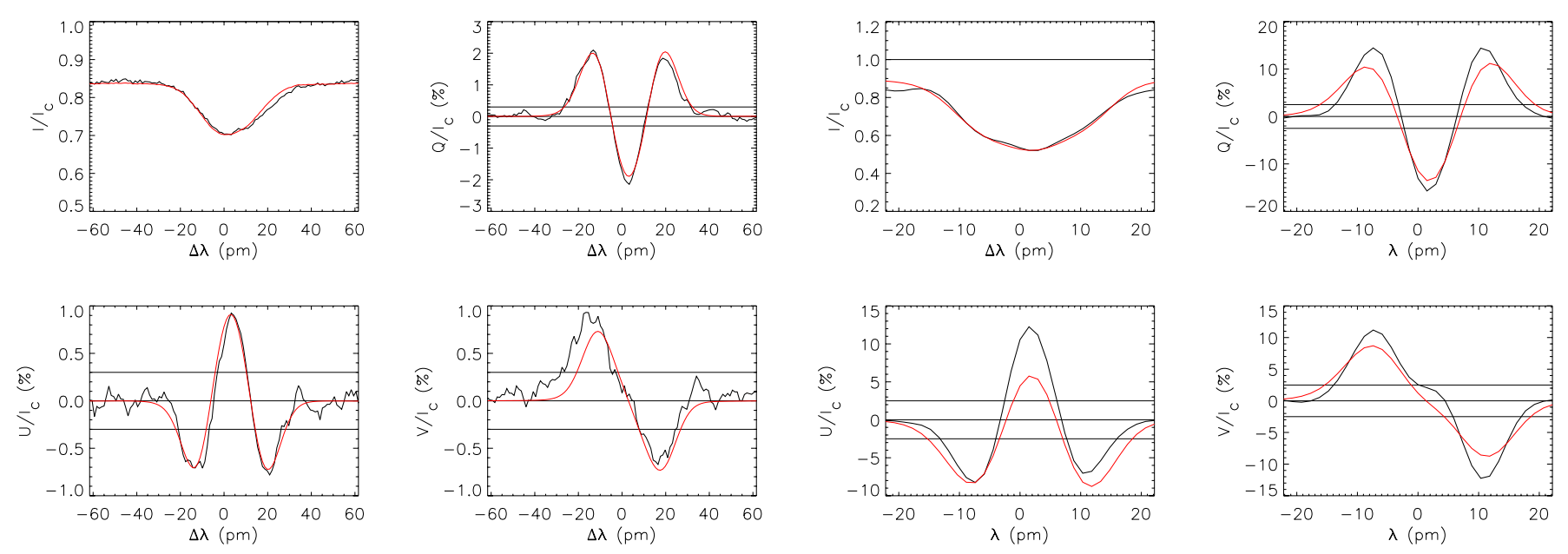

Fig. 3. Example of inversion results. The black and red curves show the observed and inverted profiles, respectively. The two sets of profiles correspond to the limb-side penumbra. Left: TIP, right: GFPI. The horizontal lines in $Q, U, V$ panels mark the zero and $\pm 3 \sigma$ noise levels. The GFPI profiles are filtered (cf. Sect. 2.2). The selected inversion setup only produces symmetric profiles, causing the imperfect fit of the e.g., Stokes- $I$ and $V$ of both instruments (Sect. 3.2).

Figure 6 shows the maps of the LRF field inclination, $\alpha$, for the selected snapshots. The azimuth maps display mostly radial field (cf. the TIP azimuth map in Fig. 7). Two ellipses that mark the light bridges (LBs) are also shown in the field strength map of TIP (bottom left panel, Fig. 4). The manual contours of the outer intensity boundary in Fig. 6 are identical with those of Fig. 5. The inner contours mark the LB boundaries. In areas outside the spot where the magnetic field strength is low (cf. Fig. 5), the amplitudes of $Q$ and $U$ are too small to provide reliable estimates for the inclination (see Borrero \& Kobel 2011).

Average values for the magnetic field inclination of umbra, penumbra, and LB are plotted in Fig. 8a versus evolution time. The evolution of the average magnetic field strength is plotted in Fig. 8b. The average inclination of the magnetic field retrieved from the TIP data is less than $10^{\circ}$ smaller (i.e., more vertical) than that of the GFPI data, both in the umbra and penumbra. Assuming that the magnetic field fans out with height, we ascribe this difference to the lower formation height of the TIP line (cf. Fig. 1). The relative large difference in the inclination of LBs between TIP and GFPI is due to the lower spatial resolution and a larger pixel size in TIP, leading to a larger contamination by umbral light. This is also seen to some extent in the magnetic field strength of the LBs in TIP data: it has a higher value, meaning that it was contaminated by umbral pixels.

\subsection{Intensity and magnetic spot boundaries}

The contours drawn in the maps of Figs. 5 and 6 are based on continuum intensity. As the maps of magnetic field strength demonstrate, kilo Gauss fields in many cases extend beyond the intensity contours. They are stronger than a typical canopy field (e.g., Rezaei et al. 2006). Towards the lower right, i.e., towards the emergence site, all evolution snapshots show strong magnetic fields of more than $1 \mathrm{kG}$. These fields come from emerging bipoles and, as discussed in the next subsection, partially merge with the spot. 

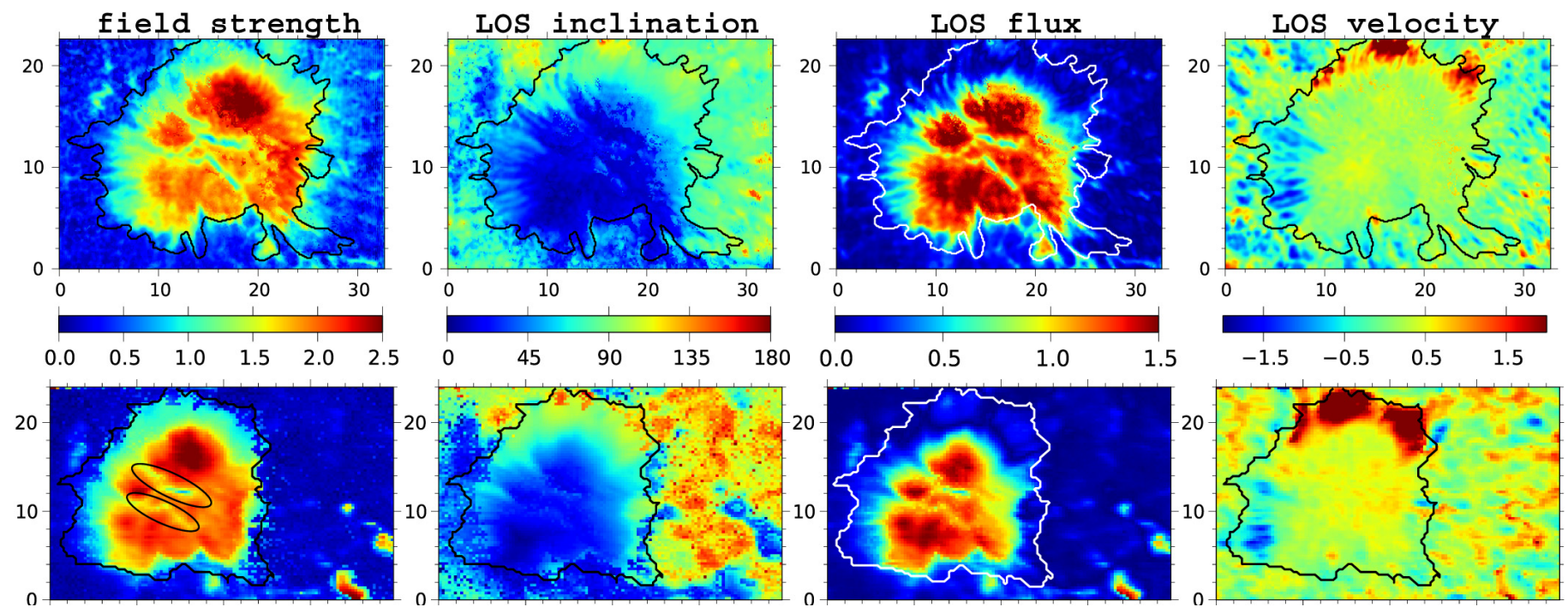

Fig. 4. Left to right: maps of the magnetic field strength (kG), LOS inclination (deg), absolute value of the LOS flux (10 ${ }^{17} \mathrm{Mx}$ ), and LOS velocity $\left(\mathrm{km} \mathrm{s}^{-1}\right.$ ) for GFPI (top) and TIP (bottom) data. For TIP the magnetic flux is scaled by a factor of 1/9 to accommodate for its larger resolution elements. The ellipses in the field strength map of TIP mark the LBs. Other parameters are like Fig. 2.

Large extensions of magnetic field beyond the intensity contours are also seen in the upper left hand side of the spot in the first three snapshots of Fig. 5, e.g., in the first map at $(x=6$, $y=15)$. As the penumbra develops on this side of the spot, these extensions become smaller. After the penumbra has formed, the intensity contours coincide well with the magnetic boundaries of the spot. That means that the magnetic flux that was initially outside the spot was incorporated into the spot during the penumbra formation process. The amount of flux, however, is only very small in the flux balance, since the field inclination is close to horizontal in these areas.

\subsection{Supply of magnetic flux from moving SFPS}

In Papers 1 and 2, we reported on the properties and processes at the flux emergence site close to the observed spot: there, elongated granules are the visible signature of flux emergence occurring on granular scales in ARs. They can be traced in maps of magnetic field strength and inclination. They have a typical size of $2-5 \mathrm{Mm} \times 0.35 \mathrm{Mm}$. Sometimes dark lanes with similar dimensions are observed (Paper 1, see also Brants \& Steenbeek 1985). Their magnetic counterparts are bipolar features with the poles being cospatial with the ends of the elongated granules (Fig. 9). As they evolve, these bipoles dissociate such that their poles separate and migrate towards their proper AR polarity. An example of such an evolution was shown in Fig. 2 of Paper 2, marked with an ellipse in Fig. 9. The LRF inclination and azimuth maps of that example, as well as the field strength and continuum intensity are seen in this figure. The field azimuth in between the opposite polarities is along the axis of the elongated granule (marked with the ellipse). The two ends of the elongated granule are also cospatial with opposite polarities as seen in the inclination panel. In the field strength map, fields of some $1.1 \mathrm{kG}$ are seen in the polarity closer to the spot, while in the other pole, the field strength amounts only to about $700 \mathrm{G}$. These small flux patches (SFPs), which are close to the forming spot and which have the spot polarity, finally merged with it. As seen in Figs. 5 and 6, e.g. $(x=20, y=8)$ or the inclination panel of Fig. 9, SFPs of both polarities exist close to the spot in the initial stages.
Using a sample of some 100 SFPs from the snapshots shown in Fig. 5, we measured a magnetic flux of $2-3 \times 10^{18} \mathrm{Mx}$ for a typical SFP, comparable to the flux values reported by Wang \& Zirin (1992). We also detect a number of SFPs that are larger than $10^{19} \mathrm{Mx}$, i.e., comparable to little pores. For comparison, the magnetic flux measured in a granule located some $20^{\prime \prime}$ away from the spot (in a direction perpendicular to the AR axis) is an order of magnitude smaller. And assuming that the average (vertical) field strength in the quiet Sun amounts to $11 \mathrm{G}$ (Lites et al. 2008), a granule with a diameter of $1^{\prime \prime}$ amounts to a flux of $4 \times 10^{16} \mathrm{Mx}$.

The magnetic field strength at the ends of elongated granules (which are in the vicinity of the spot) is typically about $1 \mathrm{kG}$ and the LRF inclination is about $70^{\circ}$. Averaging over a sample of 100 pixels in the central area of elongated granules, the field strength is $B \approx 400 \mathrm{G}(R M S=200 \mathrm{G})$, and the field inclination is horizontal within $20^{\circ}$. Similar values in magnetic field strengths have been found by Kubo et al. (2003).

As shown in the right hand panel of Fig. 9, the SFPs show a very interesting behavior when they approach the spot. They have the same azimuth as the spot, meaning that their magnetic field is aligned with field of the spot (Fig. 7) ${ }^{2}$.

\subsection{Light bridges}

Light bridges are elongated areas in the umbra with enhanced intensity (Sobotka et al. 1993). They have a fine structure consisting of granular-like segments (see Paper 1). However, the top-left end of the LB (Fig. 5) is not segmented, similar to the findings of Lites et al. (2004). LBs in the umbra typically have a width of $<2 \mathrm{Mm}$ and show up with enhanced intensity. They correspond to locations in which the magnetic field strength is significantly weaker than in the surrounding umbra, as one can see in the field strength maps of Fig. 5 (second column). They also show lower magnetic flux and more inclined fields (third and fourth columns, respectively). As seen in Fig. 5 and better in G-band and Ca images (Paper 1), LBs have a substructure: convection-like cells, and dark lanes. The dark lanes are not

2 The azimuth maps in Figs. 9 and 7 have identical color tables. 

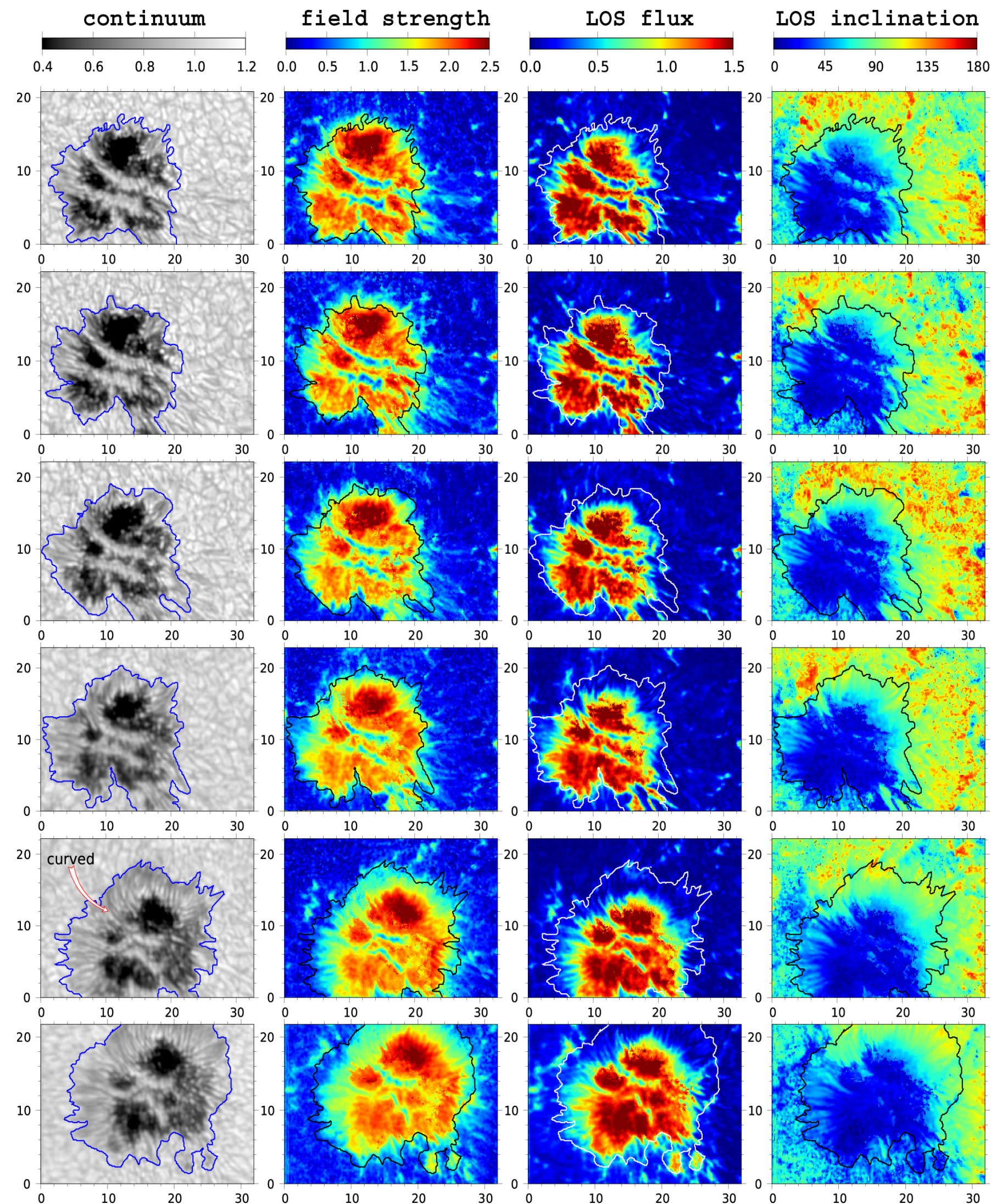

Fig. 5. Temporal evolution of the physical parameters of the spot from GFPI data. From left to right we display maps of the continuum intensity (normalized to quiet Sun), magnetic field strength (kG), LOS magnetic flux $\left(10^{17} \mathrm{Mx}\right)$, and LOS inclination (deg). From top to bottom, the maps are taken at 08:40, 08:50, 09:28, 10:13, 11:51, and 12:38 UT, respectively. The arrow in the continuum map at 11:51 points to a curved filament. Tick marks are in arcsec. 

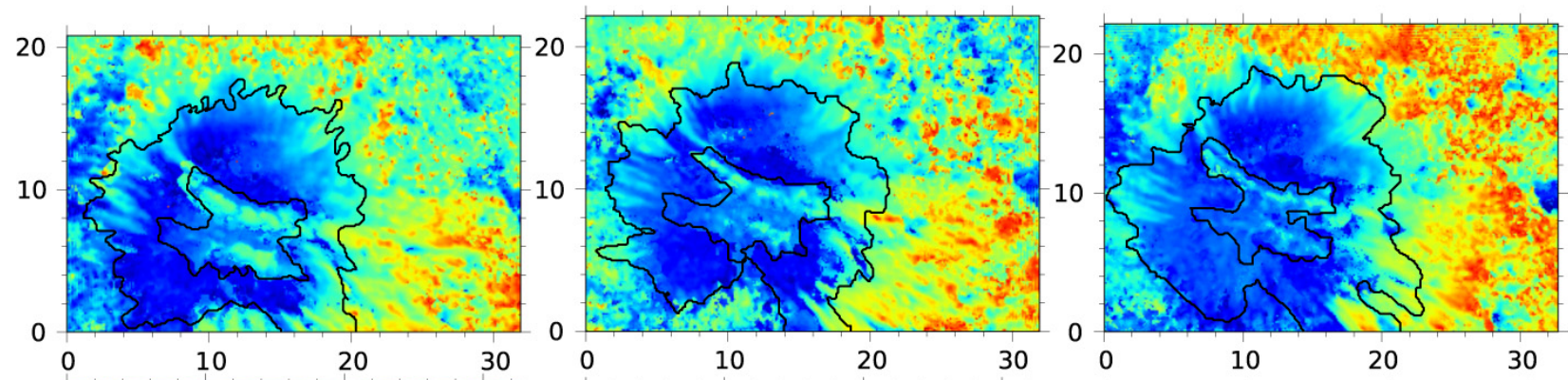

degree
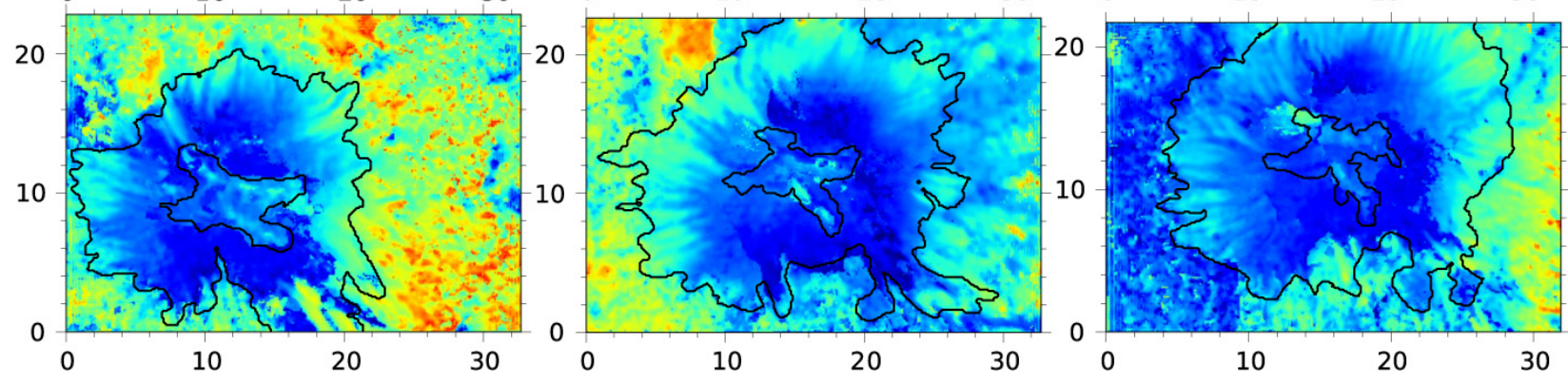

Fig. 6. Temporal evolution, as in Fig. 5, of the magnetic field inclination in the local reference frame. The maps correspond to 08:40, 08:50, 09:28, 10:13, 11:54, and 12:38 UT. The inner contours outline LBs. The outer contours are the same as in Fig. 5.

exactly located at the center of the LBs, but always towards the solar limb, being consistent with the idea that LBs are symmetric: Since the $(\tau=1)$-level is elevated relative to the surrounding umbra (Lites et al. 2004), the center side of the LB appears larger than the limb side, and the central lane is projected towards the solar limb.

Figure 10 shows the continuum intensity, magnetic field strength, and LRF inclination angle along a cut across the upper left LB (marked in the continuum map of the second top panel in Fig. 5). The cut starts on the upper right (limb side) and crosses the LB towards the lower left (center side). The LB brightness enhancement reaches up to intensity values of average granulation. The dark lane signature can be seen at the top of the intensity curve as reported earlier (Sobotka et al. 1994; Berger \& Berdyugina 2003; Giordano et al. 2008). The field lines in the dark lane are more vertical compared to the bright segments (Fig. 10). Such dark lanes show a higher contrast in Ca II K images (see Paper 1, Fig. 3). In the LB, the magnetic field strength drops below $1 \mathrm{kG}$ with a minimum value that spatially coincides with the LB dark lane. In the LRF inclination maps (Fig. 6), the LBs are outlined with contours (manual drawing based on intensity maps). The LB field lines are more inclined relative to the surrounding umbral field, reaching inclinations up to $50^{\circ}$ relative to the vertical. The azimuth map at about 11:50 UT is shown in Fig. 7. In this map, two thin ellipses mark the LBs. The field lines in the LBs are roughly aligned with the orientation of the LBs as previously reported by Louis et al. (2009).

During the sunspot evolution, the average LRF inclination in the LBs gradually decreases from $\sim 50^{\circ}$ to $\sim 37^{\circ}$; i.e., the field gradually becomes more vertical (Fig. 8a). The change in the average magnetic field strength of the LBs is shown in Fig. 8b, where it increases from 1.4 to $1.6 \mathrm{kG}$. As the magnetic properties of the LB approach those of the surrounding umbra, the LB area shrinks in the intensity images.

\subsection{Penumbra}

The development of the penumbra can be followed from the snapshots in Fig. 5 and from the G-band movie published

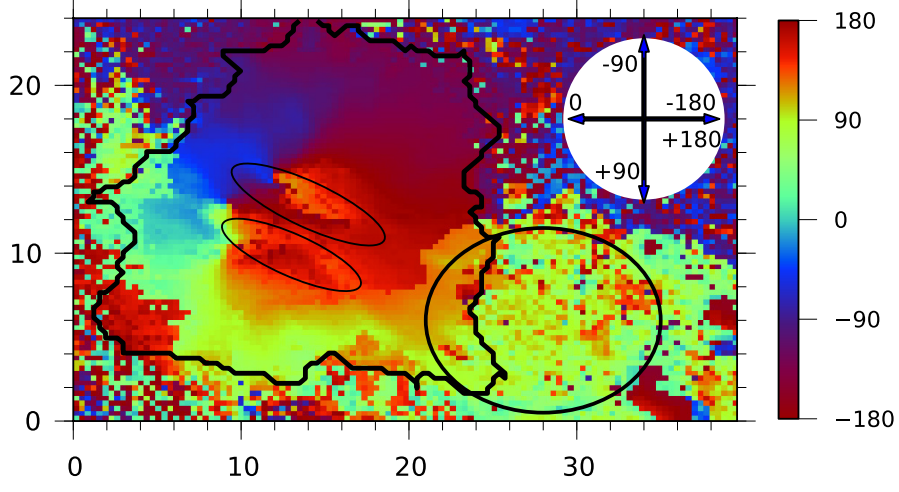

Fig. 7. LRF azimuth map in the TIP data. The corresponding maps of other quantities are shown in the bottom panels of Figs. 2 and 4. Two thin ellipses mark the LBs. The thick ellipse shows the region where SFPs arrive. Zero degree azimuth corresponds to the $(-x)$ direction, and $\pm 180^{\circ}$ correspond to the $(+x)$ direction (see arrows in the white circle). The abscissa is in arcsec.

together with Paper 1. In Paper 1 we stated that transient penumbral filaments form all around the protospot, but the locations in which stable filaments form is limited to a few sections. At the earliest stage of our observations, all penumbral sections are found next to the ends of LBs (see, e.g., $x=5, y=10$ ). The penumbral section around $(x=18, y=14)$ is not next to one of the major LBs, but as one can clearly see in Fig. 11 it is located next to an area of diffuse umbral dots, which "connect" the penumbral filaments to the major LB.

These sections grow outwards and all around the penumbra except for the lower right hand side, where only a few transient filaments form. There it seems that the emerging flux and the approaching SFPs inhibit the formation of stable filaments. This indicates that, for the penumbra to form, a quiet surrounding in which the penumbra can expand and evolve is necessary.

While the penumbra develops, the average penumbral magnetic field strength keeps constant around $1500 \mathrm{G}$ (Fig. 8b), showing an insignificant decrease $(\delta B \sim 100 \mathrm{G})$. The average 

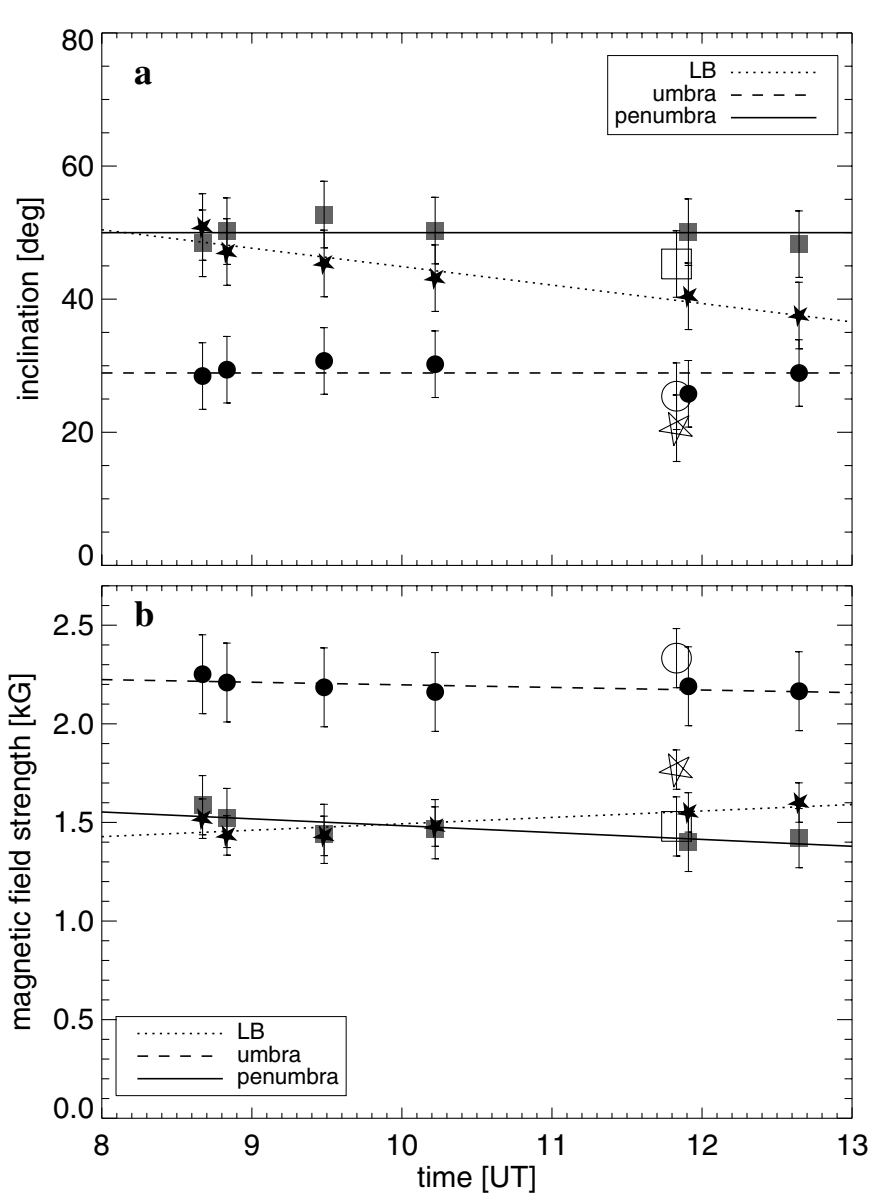

Fig. 8. Variation in the LRF inclination angle (top) and magnetic field strength (bottom) in the umbra (dashed, circle), penumbra (solid, square), and LBs (dotted, asterisk). The corresponding TIP values are shown as empty symbols. The time steps are the ones shown in Fig. 6.

LRF inclination also stays roughly constant at $\sim 50^{\circ}\left(\delta \theta \sim 5^{\circ}\right.$, see Fig. 8a). These are typical values for the penumbrae (Lites \& Skumanich 1990; Keppens \& Martinez Pillet 1996; Mathew et al. 2003; Bellot Rubio 2004; Beck 2008; Tritschler 2009). On the border between the penumbra and quiet Sun, the average field strength decreases from $1000 \mathrm{G}$ to $800 \mathrm{G}$.

The filamentary structure of the penumbra can be seen in both intensity and magnetic maps. The magnetic field inclination, both in the LOS and LRF frames (Figs. 5 and 6), shows clear signs of spine and interspine structures (Lites et al. 1993; Sánchez Cuberes et al. 2005). The peak-to-peak difference in the LRF inclination between spines and interspines is about $10^{\circ}-20^{\circ}$. This variation is smaller than the corresponding values for a mature spot (e.g., Kubo et al. 2007). However, such a peak-to-peak difference is a function of radial distance from the spot center (Westendorp Plaza et al. 2001).

In the existing penumbral sections for the snapshot at 08:40 UT at around ( $x=4, y=10)$, the LRF inclination increases radially from $40^{\circ}$ (inner penumbra) to $65^{\circ}$ (outer penumbra) and at $(x=16, y=12)$ from $40^{\circ}$ to $70^{\circ}$, and keeps nearly constant with time. In contrast, the inclination increases with time as the penumbra advances in the new forming section, e.g. around $(x=12, y=18)$ in the snapshot at 08:40 UT

We aligned the larger part of umbra in the first (08:40 UT) and last (12:38 UT) stages (Fig. 5). While the field lines become more inclined with the developing penumbra, we find that the magnetic neutral line keeps the same distance to the umbra.

\subsection{Curved penumbral filaments}

At late stages, the upper penumbra shows filaments that are not radial but curved (Fig. 5). The curvature, as seen in the continuum and G-band images, amounts to more than $45^{\circ}$. Beside that, as seen in the bottom middle panel of Fig. $6(x=16, y=22)$, the field lines change their polarity in that location. From other snapshots not shown here, we find that there are kilo Gauss fields outside the spot and close to these curved area. Parts of this opposite-polarity area coincide with an enhanced brightening in the cotemporal $\mathrm{Ca}$ II $\mathrm{K}$ image. Reported amounts of curvature in the penumbral filaments are usually less (Lites \& Skumanich 1990; Keppens \& Martinez Pillet 1996; Westendorp Plaza et al. 2001). Gurman \& House (1981), however, reported a twist angle of $35^{\circ}$. From the maps of the same spot in the later days (Paper 1, Fig. 2), we know that the curved filaments become essentially radial in subsequent days.

\subsection{Umbra}

Figure $8 \mathrm{~b}$ shows the evolution of the umbral magnetic field strength, which stays constant at $2.2 \mathrm{kG}$ during the evolution. The maximum field strength - determined as the average of 100 umbral pixels that show the strongest field strength values also stays constant at $\sim 2.7 \pm 0.2 \mathrm{kG}$ (not shown). The average of the umbral inclinations in the LRF remains constant at some $28^{\circ}$. However, the inclination in the umbra is not uniform, especially in the lower part. There, at the border between the umbra and quiet Sun the inclination is less than $10^{\circ}$ and increases gradually from there toward the spot center.

Another important finding is that the small umbra in the left hand section shrinks in size, while its mean inclination increases from $30^{\circ}$ to $40^{\circ}$. In the meantime, a new umbral core develops towards the emergence region, where no penumbra forms. Thus, the net umbral area remains constant during the spot's evolution (Paper 1).

Figure 12 shows the variation in the total magnetic flux with time, both in the LOS and LRF frames. As expected, the values are higher in the LRF. The magnetic flux of the penumbra is larger than the flux in the umbra (or umbra + LBs), already from early stages. That the majority of the magnetic flux is in the penumbra is a universal property of sunspots (Schmidt 1991; Solanki \& Schmidt 1993; Balthasar \& Collados 2005), and suggests that the penumbra is not flat, but extends into deep subphotospheric layers.

\subsection{Flux budget}

Using the G-band and Ca II K time series (Paper 1), we have reported that the umbral area stays constant $\left(100 \operatorname{arcsec}^{2}\right)$ while the total sunspot area increases from 230 to $360 \operatorname{arcsec}^{2}$ (an increase of 56\%). That means the penumbral area increased from 130 to $260 \mathrm{arcsec}^{2}$, corresponding to an increase of $100 \%$. This increase in area reflects the increase in magnetic flux. Here, we determine the increase in the magnetic flux, using the magnetic field strength, $B$, and the inclination angle in the LOS frame, $\gamma$, or in the LRF, $\alpha$ (Sect. 3):

$\Phi=A B \cos \alpha$

where $A$ is the deprojected area of each pixel. 

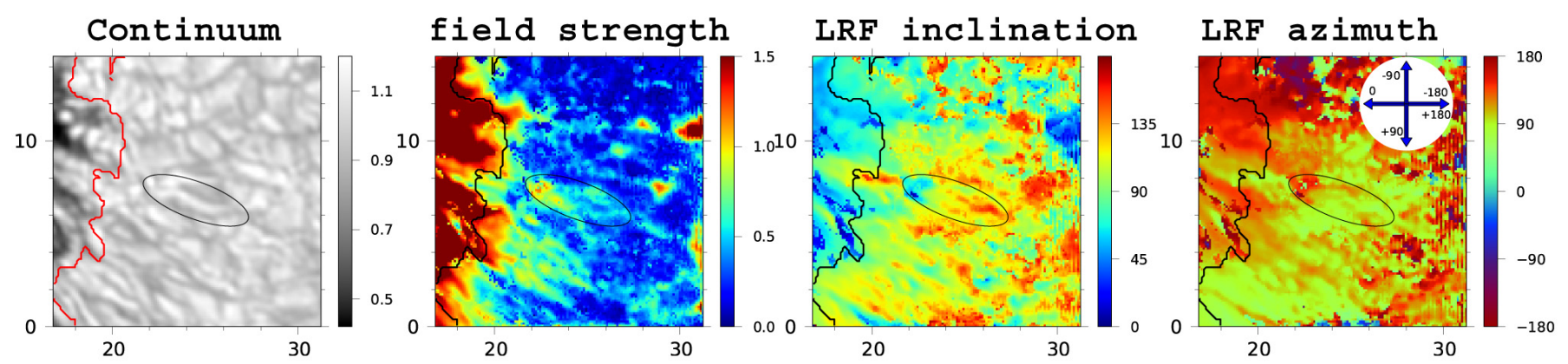

Fig. 9. Elongated granule and the corresponding bipole (marked by ellipse) in GFPI data. Left to right: continuum intensity (normalized to quiet Sun), magnetic field strength between zero and $1.5 \mathrm{kG}$, inclination, and azimuth (deg) in the local reference frame. The coordinates of the selected area shown here correspond to the middle-top panel of Fig. 6.

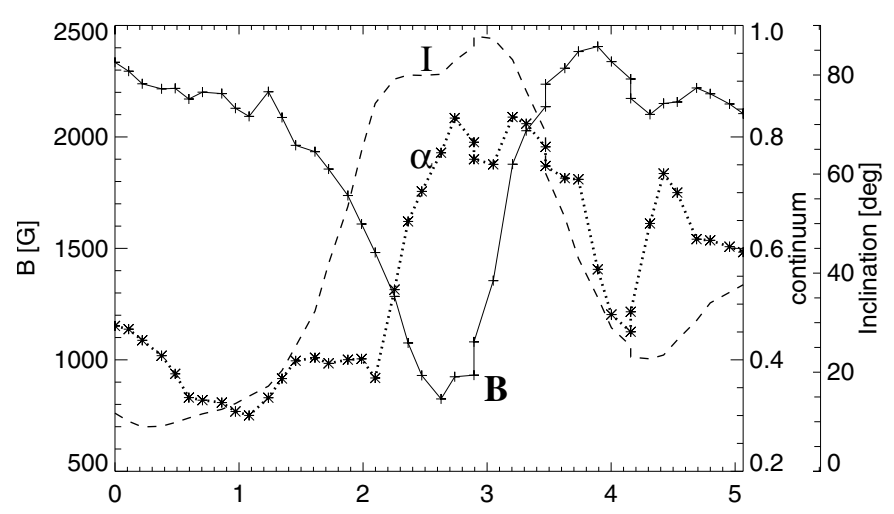

Fig. 10. Variation in the continuum intensity (dashed), magnetic field strength (solid), and inclination (dotted) across the upper left LB in the second top panel of Fig. 5. The abscissa is distance in arcsec, and the cut starts at the upper right of the LB.

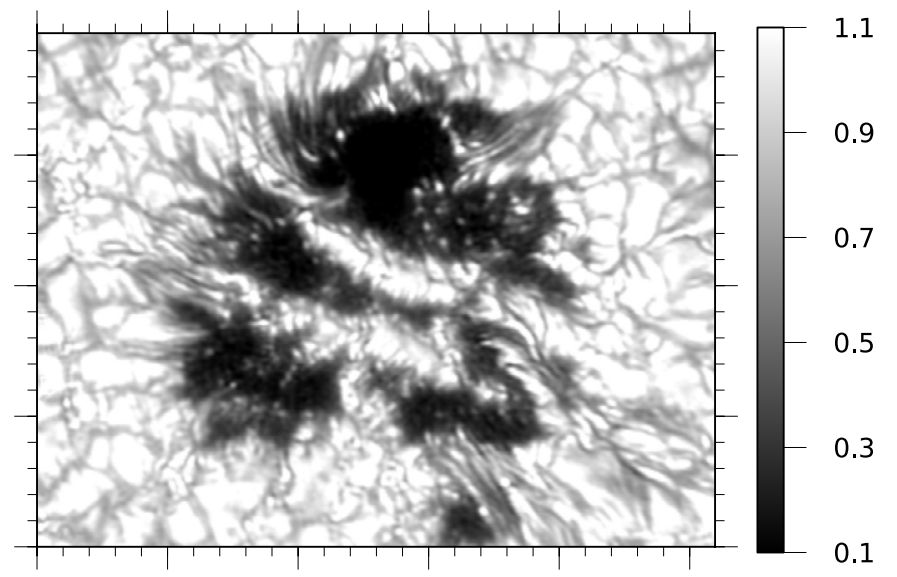

Fig. 11. G-band snapshot of the forming sunspot at 08:33 UT. The intensity has been clipped to lie between 0.1 and 1.1 (1.0 is the average quiet Sun). Each tick mark is one arc second.

The umbral area was defined as the area of dark pixels with a continuum intensity lower than $0.5 I_{\mathrm{c}}$. For the penumbra and LBs, we used manual contours. The penumbral contours are shown in Fig. 5, while both penumbra and LBs contours are shown in Fig. 6. As mentioned before, the average field strength of the umbra is constant. Considering that the umbral area keeps constant (Paper 1, Fig. 4), we find that the total umbral flux is
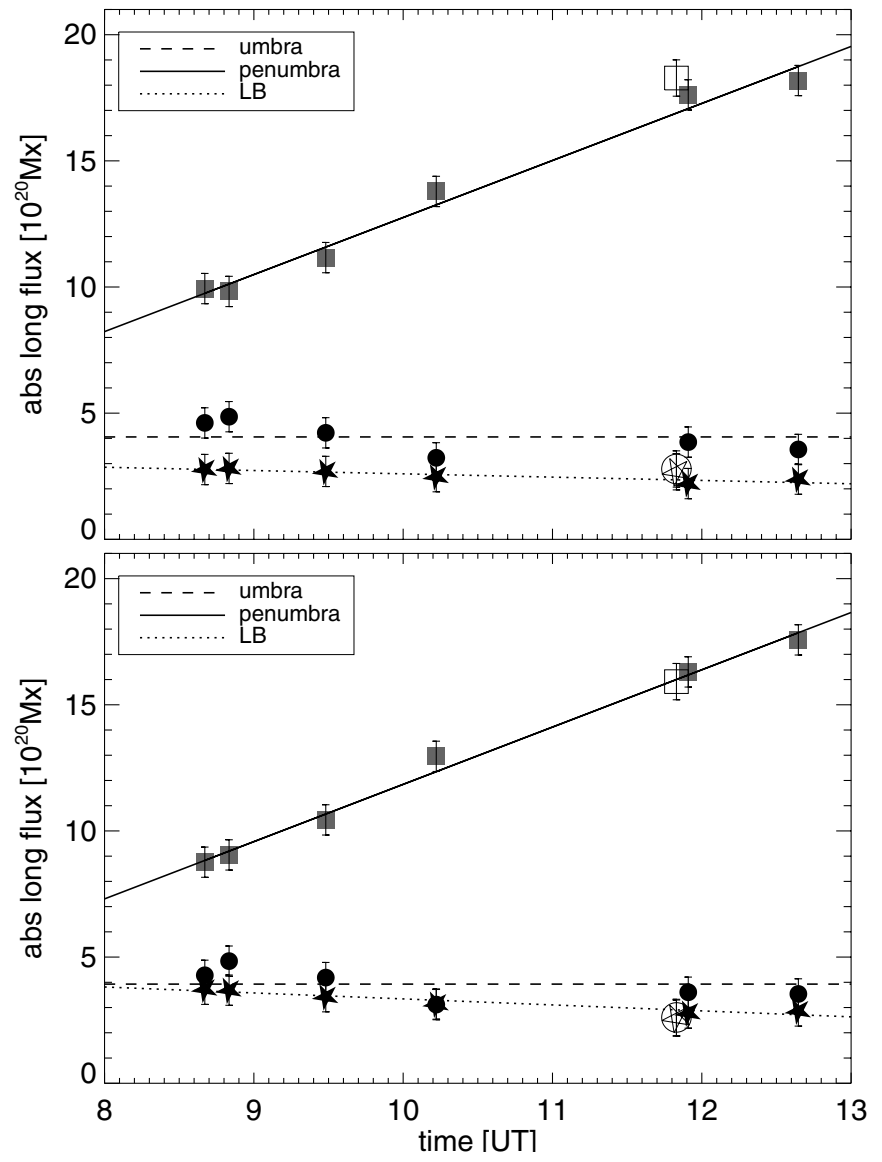

Fig. 12. Similar to Fig. 8 but for the magnetic flux. Top: LRF, bottom: LOS frame.

constant within our errors. There is a slight tendency of a decreasing umbral flux as is the case for the area (Paper 1).

The umbrae show strong dynamics during the sunspot evolution. For instance, parts of the LBs dissolve into umbral dots and diffused emission, while parts of the umbra shrink at expenses of the penumbral formation (left umbrae) and other umbral subareas change in size. However, the overall result is that the umbral area, field strength, and flux stay constant.

As mentioned above, the sunspot (penumbral) area increased by about $56 \%(100 \%)$. We find that the penumbral flux increases 
from $9.7 \times 10^{20}$ to $18.2 \times 10^{20} \mathrm{Mx}(88 \%)^{3}$. The void symbols in Fig. 12 represent the corresponding values for the TIP map shown in Fig. $2^{4}$.

\subsection{Summary of results}

We now summarize the magnetic properties of the forming spot presented in the previous sections.

- A strong $(\approx 1 \mathrm{kG})$ inclined $\left(45^{\circ}-60^{\circ}\right)$ magnetic field component is present outside the visible spot boundaries previous to the penumbra formation.

- The maximum $(\sim 2.7 \mathrm{kG})$ and the spatially averaged $(2.2 \mathrm{kG})$ magnetic field strength of the umbra, stays constant during the penumbra formation. In addition, the penumbral field strength slightly decreases around $1.5 \mathrm{kG}$, and in the LB it increases from $1.4 \mathrm{kG}$ to $1.6 \mathrm{kG}$ (Fig. $8 \mathrm{~b}$ ).

- The average LRF magnetic field inclination in the umbra $\left(28^{\circ}\right)$ and penumbra $\left(50^{\circ}\right)$ do not change during the spot formation, while in the LBs it decreases from $50^{\circ}$ to $37^{\circ}$; i.e., the field lines in the LBs become more vertical (Fig. 8a).

- The total magnetic flux of the spot increased by $40 \%$, from $17.4 \times 10^{20}$ to $24.2 \times 10^{20} \mathrm{Mx}$. This is consistent with the earlier report of a $56 \%$ increase in the spot area (Paper 1). The increase in the penumbral area is of about $100 \%$, corresponding to an increase of the magnetic flux of $88 \%$ (Fig. 12). While the flux in the umbra keeps constant, the magnetic flux of the LBs decreased from $2.8 \times 10^{20}$ to $2.4 \times 10^{20} \mathrm{Mx}$ (its area shrinks).

- Averaging over some 100 SFPs, we find that the mean magnetic flux of individual SFPs amounts to $2-3 \times 10^{18} \mathrm{Mx}$. The typical value of the inclination of the field lines in SFPs close to the spot is about $70^{\circ}$.

- The average rate of flux accumulation in the spot is $4.2 \times$ $10^{16} \mathrm{Mx} \mathrm{s}^{-1}$, which is equal to the merge of one SFP per minute to the spot.

- The new magnetic field lines reach the spot boundary with the same azimuth angle as the spot azimuth; i.e., both SFP and spot field lines are coaligned.

- The new flux arrives in the side of the spot facing the AR opposite polarity, while the penumbra develops on the other side of the spot. During this process, the position of the magnetic neutral line relative to the umbra does not change.

- The first penumbral sections preferably form close to the ends of LBs. As the penumbral sections form, the inclination is increasing.

\section{Discussion}

In this section we attempt to construct a consistent scenario of the spot formation process by assembling our results with previous findings. After sorting out the involved timescales (Sect. 5.1), we discuss the properties of the emergence site and the transport of flux to the spot (Sects. 5.2 and 5.3). We ascribe a crucial role to LBs, as remnants of trapped granules between merging pores. They ignite the formation of the penumbra and allow flux to be transported from one side of the spot to the other (Sects. 5.4, 5.5, and 5.6). In the last section we elaborate on the

\footnotetext{
3 The last snapshot does not fully cover the spot (Fig. 5), so the true amount of the flux is a few percent more.

4 The spatial sampling in the TIP data is larger than GFPI, so there is more uncertainty in the manual contours, leading to larger uncertainties in the total magnetic flux.
}

necessities to form a penumbra and discuss the properties of the developing penumbra.

\subsection{Timescales in a sunspot}

Sunspots live for days to weeks (Bray \& Loughhead 1964). Next to the long lifetime $\left(t_{1} \sim a\right.$ week), there is a formation timescale. This is the time required to assemble a spot out of disorganized emerged flux. We estimate the formation time to be about $t_{\mathrm{f}} \sim 0.5$ day. The dynamical timescale is the time that the spot requires to rearrange the equilibrium configuration of the field lines in a smooth transition between two equilibrium states. The dynamical time corresponds to the Alfvén travel time across the spot. The Alfvén and sound travel speeds (at $\tau=1$ ) are about $v_{\mathrm{A}} \sim 8 \mathrm{~km} \mathrm{~s}^{-1}, c_{\mathrm{s}} \sim 6 \mathrm{~km} \mathrm{~s}^{-1}$, respectively (Schlichenmaier 1997). The travel time in our sunspot with $d \sim 15 \mathrm{Mm}$ is $t_{\mathrm{d}} \sim 1 \mathrm{~h}$. Individual penumbral filaments also form on a dynamical timescale. Smaller timescales, $t_{\mathrm{s}} \lesssim 5 \mathrm{~min}$, have been observed by Sobotka et al. (1997, their Fig. 5) and Ortiz et al. (2010) in umbral dots and LBs. In summary the timescales,

$$
t_{1} \gg t_{\mathrm{f}} \gg t_{\mathrm{d}} \gg t_{\mathrm{s}}
$$

correspond to different physical phenomena: the decay process, the integration of the new flux, the penumbral formation, and probably some type of convective timescale, respectively.

\subsection{Elongated granules}

The AR flux emergence site close to the leading spot is characterized by elongated granules and extended dark lanes, together with micropores (circular patches of reduced intensity) as seen in intensity (see Paper 1 and e.g., Gömöry et al. 2010). The properties of the elongated granules found in our data are similar to those observed by Brants \& Steenbeek (1985) in a forming AR, by Strous \& Zwaan (1999) in a developing AR, and by Otsuji et al. (2007) in the moat of a fully fledged sunspot. They are also found in numerical simulations of flux emergence in ephemeral (Cheung et al. 2007, 2008) and active (Cheung et al. 2010) regions.

The magnetic field strength in the emergence site is weaker than in the vicinity of the spot (see Sect. 4.2). Some magnetic field intensification must then work on the $(\mathrm{hG})$ emerged flux to facilitate the kilo Gauss field strength observed at the ends of elongated granules (e.g., Weiss 1966; Spruit 1979; GrossmannDoerth et al. 1998). These mechanisms have some observational support (Bellot Rubio et al. 2001; Nagata et al. 2008; Danilovic et al. 2010).

In our observations, elongated granules appear only in a limited cone close to the spot toward the opposite AR polarity $(\sim \mathrm{a}$ quadrant). In contrast, the simulation by Cheung et al. (2010) shows elongated granules almost isotropically around the spot. This might be due to the limited separation of the two polarities in the simulation domain and possibly an effect of periodic boundary conditions.

Elongated granules carry significant amounts of magnetic flux, but have similar continuum intensities compared to the quiet Sun (cf. Leka \& Skumanich 1998, who call them radiatively undisturbed points). On the opposite ends of some elongated granules the magnetic field has opposite polarities. To understand the overall field topology, the orientations of these bipoles is crucial. In a simple model, the SFPs having the same polarity as the spot finally merge with it. However, observations demonstrate that the topology is typically more complex 
(e.g., Bernasconi et al. 2002; Pariat et al. 2004). As explanations, undulations of field lines are discussed as due to their interactions with the granular convection and resistive effects like magnetic reconnection (e.g., Strous \& Zwaan 1999; Cheung et al. 2008). We find elongated granules with field lines of opposite polarity at either end, but our field of view and the temporal resolution are insufficient to reconstruct the evolution of the field topology of our emergence site.

\subsection{Flux transport in the active region}

On July 4, at 08:30 UT the protospot had a flux of $17.4 \times 10^{20} \mathrm{Mx}$. In $4.5 \mathrm{~h}$, it transformed into a mature spot with a major penumbra. Two questions arise. (1) How did the initial protospot form? (2) How did the penumbra grow in size and in magnetic flux?

(1) Merging pores: From SoHO/MDI data it is seen that two pores appeared next to each other at 20:47 UT on July 3 (Schlichenmaier et al. 2011). These pores increase in size, merge, and form the protospot, in accordance to the proposal of Zwaan (1992).

(2) SFPs supply flux: In the $4.5 \mathrm{~h}$ of the VTT observation no further pore merges with the spot. Although pores are observed to merge later again, the flux increase by $\sim 7 \times 10^{20} \mathrm{Mx}$ between 08:30 UT and 13:00 UT cannot be due to merging pores. The tiny pore below the spot is included in our contours, i.e., merged before 08:30. The increase in the spot's flux is comparable to the flux of a few pores. Since we see examples of SFPs that merge with the spot, we propose that all the additional flux was carried to the spot by SFPs. Although the coalescence of pores (Zwaan 1992) is therefore an important step in the formation of protospots (initial phase), a majority of the extra flux required to form the penumbra is provided via SFPs. In our case, an increase rate of $4.2 \times 10^{16} \mathrm{Mx} \mathrm{s}^{-1}$ needs to be supplied by merging SFPs; i.e., one SFP per minute is needed.

We can also compute a value for the flux increase rate for forming the protospot. The flux emergence started latest on July 3, at 20:47 UT (see Schlichenmaier et al. 2011, using MDI data). Using the known value of the flux of the protospot, we estimate that the initial flux rate amounts to $3.9 \times 10^{16} \mathrm{Mx} \mathrm{s}^{-1}$. These two rate values agree with each other and with previous observations (e.g., Kubo et al. 2003) and comparable to the values of Cheung et al. (2010, their Fig. 10) from an intermediate phase in the simulation. For a pore that develops a partial penumbra Leka $\&$ Skumanich (1998) give a lower rate of $1 \times 10^{16} \mathrm{Mx} \mathrm{s}^{-1}$.

From these considerations, we presume that pores also grow by merging SFPs. In that sense, elongated granules, emerging bipoles, and the corresponding SFPs are the building blocks of large-scale magnetic structures in the photosphere. SFPs build up pores, and then spots are formed out of pores and SFPs.

\subsection{Light bridges as remnants of spot formation}

During the process of merging pores, a LB forms as the granular area in between the pores that are "trapped". Using SoHO/MDI data (Schlichenmaier et al. 2011), we find that at least two pores of the same polarity emerged close to each other on July 3, and merged to form the proto-spot on July 4. In that sense, the LBs that we observe on July 4 are remnants of the formation process of the protospot. A light bridge that remains after a pore has merged can also be seen on July 5 (see Fig. 2 in Paper 1, and also Rouppe van der Voort et al. 2010). During the later evolutionary stages other types of LBs exist (e.g., Katsukawa et al. 2007). Garcia de La Rosa (1987) proposes that LBs that form in the decaying phase are related to the ones that faded away in the forming phase.

As seen in Sect. 4.3, LBs have a weaker and more inclined magnetic field than the umbra. Such a reduced magnetic field strength agree with Rüedi et al. (1995) and Leka (1997). We estimate that the horizontal gradients of the magnetic field strength in the LBs is about $1 \mathrm{G} \mathrm{km}^{-1}$, consistent with the values reported by Shimizu et al. (2009). This is more than what Leka (1997) finds on average, most probably, since our spatial resolution is significantly better.

As described in Paper 1, a chain of elongated segments forms a bright lane within a LB. That these segments are smaller than granules in the quiet Sun is expected to be the result of the magnetic field that alters the mode of convection (e.g., Rimmele 1997; Schüssler \& Vögler 2006; Bharti et al. 2009; Scharmer 2009). The mere existence of these bright lanes provides evidence of overturning magnetoconvection in the LB.

During the sequence of our observation, the LB area becomes smaller and observations from July 6 show that the LBs have disappeared. This means that the initially weakly magnetized plasma gradually becomes magnetized and the field strength increases, thereby further suppressing the regular type of convection. In other words, the magnetic field gradually intrudes into the gap of weak field. During this evolution the LB transforms into an area of umbral dots and diffused emission, and finally fades away into an umbral area.

\subsection{Light bridges as ignitor for penumbra formation}

Although LBs fade away as the spot develops, they seem to play a crucial role in the penumbra formation process. We find that the first penumbral filaments form in the vicinity of LBs. This agrees with observations by Yang et al. (2003). There are more examples where the first penumbral filaments form as extensions of LBs, as seen in the DOT archive (Rutten et al. 2004, e.g., spot of July 13, 2005).

However, this property does not apply to areas facing the opposite AR polarity. No stable penumbra form on these ends of the LBs. We ascribe this to the ongoing activity and surmise that the presence of magnetic field in the emergence site hinders the magnetic field of the protospot to spread out.

On the side opposite to the emergence site, the magnetic field has space to unfold, to become more inclined and to form a penumbra. That this happens at the ends of LBs could indicate that the transition in the penumbral mode of convection starts where the field strength is reduced. The effect of granular motions onto the magnetic fields should be strongest at locations of the weak magnetic field, and it seems plausible that this should be the locations where the penumbra forms first. However, from our observation we cannot characterize this transition further.

It has been suggested by Weiss et al. (2004) that field lines are dragged downwards by granular motions (see also Wentzel 1992). Thereby the field inclination increases and a penumbra forms. In their scenario this happens at corrugations of the magnetopause, which are caused by the fluting instability (e.g., Schüssler 1984). Inspired by our observation, we speculate that such a process would preferably occur where the field is weak, i.e. only at the ends of LBs. There it could also be the fluting instability that initiates the transition from LB into penumbra, but neither our observations nor the model calculations of Weiss et al. (2004) allow further insight. 


\subsection{Light bridges as channels for magnetic flux}

The LBs may yet have an additional key-role in transforming a proto-spot without penumbra into a spot with penumbra: they are the channels in which the magnetic flux is transported from the one side of the spot to the other. This results from the following considerations:

From the temporal evolution we see that SFPs that migrate towards the spot tend to merge the spot near the right end of the upper LB (cf. Fig. 5). We have presented evidence that the increase in magnetic flux of the evolving spot comes from the flux that is added by SFPs. In this sense, LBs seem to be the entry gate for a new flux that merges the spot. Since the penumbra forms on the other side, while the magnetic neutral line relative to the umbra does not change its location, magnetic flux must be transported through the spot, from one side to the other. And since the new flux enters the spot in the LB and the penumbra starts to form on the other side of the LB, it seems plausible to assume that the flux is transported through the LB and not through the areas of strong fields. We envisage two processes that could accomplish such a transport of flux:

(1) Advection of flux: in the first possibility, a stream of plasma advects the flux elements from one side to the other. This seems possible because it would take some two hours to cross the LB if the flow has a speed of $1 \mathrm{~km} \mathrm{~s}^{-1}$ (Hirzberger et al. 2002; Louis et al. 2008). Visual inspection of our G-band movie supports the presence of such a flow: Proper motions are seen that could be a signature of shear flows in the LB (Bello González et al. 2011).

(2) Reconfiguration: for this process we assume that the spot is in quasi-stationary equilibrium at all times during its growth phase, since the dynamical timescale is less than one hour. We envisage that the spot evolves through a sequence of quasistationary equilibria. When new magnetic flux joins the spot (a SFP merges), the magnetic field configuration is perturbed and the spot is out of balance. This perturbation causes a perturbation in magnetic field strength and hence in magnetic pressure. This pressure perturbation, which consists of a compression of field lines, propagates through the spot with Alfvén speed and brings the spot back into magnetostatic equilibrium. In this way, magnetic flux is redistributed within the spot. Thereby, this process could explain the increase in magnetic flux beyond the magnetic neutral line. These waves of magnetic pressure variations would preferably propagate through the LBs, since there the field strength is lower and less energy is needed for the field compression than in the umbra.

If one such a compression wave reaches the other side, the spot has grown in magnetic flux and is in a new equilibrium. In this new configuration the outermost field lines are more inclined, which potentially favors the formation of a penumbra.

Both these processes could exist in parallel, and both would favor the end of LBs as the locations where the formation of the penumbra starts. In the further evolution, when the flow through the LBs has ceased and when the field strength in LB has increases, only the "reconfiguration" process remains. Then the pressure perturbations travel through the umbra, and the segments where no penumbra has yet formed can be filled.

\subsection{Some properties of the developing penumbra}

Wherever and whenever continuum and flow maps show the signatures of a penumbra, the magnetic field has the typical properties of a penumbra: a strength of some $1.5 \mathrm{kG}$ and a mean inclination of some $50^{\circ}$. However, at the earliest stages, the magnetic area of the protospot extends over the visible limits. There the field strength is also more than $1 \mathrm{kG}$, and the field has large inclinations, but neither continuum maps nor the flow maps show a sign of a penumbra. During the course of our time series, these areas are mostly converted into penumbral areas. Unfortunately, we have no explanation for why there is flux in the early stages on the side opposite to the emergence site, which is not yet integrated into the visible boundaries of the spot.

In Sect. 4.5 we report on the presence of opposite polarities at the outer end of the curved filaments. The presence of these opposite polarities during the formation could be interpreted as the turbulent pumping process that is described in Weiss et al. (2004): field lines that reach some critical angle of inclination, which are grabbed by convective plumes and dragged downwards. This could explain the opposite polarity at the boundary of the spot as the footpoints of returning field lines.

\subsection{When does a penumbra form?}

Last but not least, we want to discuss the question about the conditions that are needed to form a penumbra. The first condition is a minimum of magnetic flux. According to Zwaan (1987), a flux of at least about $5 \times 10^{20} \mathrm{Mx}$ is required to assemble a spot. This is consistent with the result of Bogdan et al. (1988) who note that the minimum umbral radius in a large sample of spots is about $1.5 \mathrm{Mm}$ (assuming that the radial extension of the penumbra is that of the umbra and plugging in typical values for the field strength). Leka \& Skumanich (1998) observed a sunspot that had a partial penumbra with a magnetic flux of $1 \times 10^{20} \mathrm{Mx}$, comparable to critical flux of $1-7 \times 10^{20} \mathrm{Mx}$ reported by Rucklidge et al. (1995). Therefore, various authors come to the comparable results for the critical flux: $\phi_{\text {crit }} \lesssim 5 \times 10^{20} \mathrm{Mx}$. The protospot in our data is clearly larger with a magnetic flux of $17 \times 10^{20} \mathrm{Mx}$.

In addition, the properties of the magnetic field certainly pose necessary conditions: As seen in the top rows of Fig. 5, we find for the critical magnetic field strength, $B_{\text {crit }} \lesssim 1.6 \mathrm{kG}$. From a comparison of the maps of continuum (Fig. 5) and inclination (Fig. 6), we find that the existence of a penumbral filament is associated with large inclination angles, $\alpha_{\text {crit }} \gtrsim 60^{\circ}$. These critical values imply that the spot is growing, and its flux is increasing such that the field strength and inclination become critical.

Moreover, we find that stable filaments only form away from the emergence site. Therefore, it seems necessary to have a "quiet" environment into which the penumbra can grow. These are conditions that are fulfilled in the forming penumbra of our data set, but we cannot tell whether they are sufficient.

\section{Conclusion}

We present the spectropolarimetric data of an emerging active region and a forming penumbra. The emergence site is characterized by elongated granules that are associated with small flux patches (SFPs) of opposite polarities. These SFPs coalesce to form pores and spots. Spots partly form from merging pores, but a substantial fraction of the flux also comes from merging SFPs. As a result, SFPs are the building blocks of structure formation in ARs.

During the forming phase, LBs are the remnants of merging pores and they play a key role in the formation process. LBs provide the channels through which the flux can be transported from the emergence side to the other side of the spot, where the 
penumbra forms. This transport could be accomplished (a) by a flow that advects the flux or (b) by readjustments of the magnetic field configuration to accommodate the increasing flux, and the associated waves of magnetic pressure variation favor propagating in LBs. At the same time, LBs are the ignitors for the formation of penumbra filaments as the penumbra starts to form next to the ends of LBs.

The formation of the penumbra is linked to a set of conditions as there are critical values for the magnetic flux of the protospot, for the field strength, and for the inclination angle of the field. In presence of these conditions and a "quiet" magnetic surrounding, individual stable filaments form on a dynamical timescale.

Acknowledgements. The German VTT is operated by the Kiepenheuer-Institut für Sonnenphysik at the Spanish Observatorio del Teide. The success of the observational setup was achieved by the common effort of members of the institutes involved in the development of the German solar observatories (IAP, IAC, MPS, KIS). We are grateful to Bruce Lites for assisting us with the AZAM code. We wish to thank Christian Beck for helpful suggestions. NBG acknowledges financial support by the DFG grant Schm 1168/9-1. R.S. acknowledges fruitful discussions at the workshop on "Filamentary Structure and Dynamics of Solar Magnetic Fields" at the ISSI in Bern.

\section{References}

Abbett, W. P., Fisher, G. H., \& Fan, Y. 2000, ApJ, 540, 548

Allende Prieto, C., Asplund, M., \& Fabiani Bendicho, P. 2004, A\&A, 423, 1109 Balthasar, H., \& Collados, M. 2005, A\&A, 429, 705

Beck, C. 2011, A\&A, 525, A133

Beck, C., Schlichenmaier, R., Collados, M., Bellot Rubio, L., \& Kentischer, T. 2005a, A\&A, 443, 1047

Beck, C., Schmidt, W., Kentischer, T., \& Elmore, D. 2005b, A\&A, 437, 1159

Beck, C. 2006, Ph.D. Thesis, Albert-Ludwigs-University, Freiburg

Beck, C. 2008, A\&A, 480, 825

Beck, C., Mikurda, K., Bellot Rubio, L. R., Kentischer, T., \& Collados, M. 2007, in Modern solar facilities - advanced solar science, ed. F. Kneer, K. G. Puschmann, \& A. D. Wittmann, 55

Beck, C. A. R., \& Rezaei, R. 2011, A\&A, 531, A173

Beck, C., Rezaei, R., \& Fabbian, D. 2011, A\&A, 535, A129

Bellot Rubio, L. R. 2003, Inversion of Stokes profiles with SIR (Freiburg: Kiepenheuer Institut für Sonnenphysik)

Bellot Rubio, L. R. 2004, in Reviews in Modern Astronomy, ed. R. E. Schielicke, 17,21

Bellot Rubio, L. R., Rodríguez Hidalgo, I., Collados, M., Khomenko, E., \& Ruiz Cobo, B. 2001, ApJ, 560, 1010

Bellot Rubio, L. R., Balthasar, H., Collados, M., \& Schlichenmaier, R. 2003, A\&A, 403, L47

Bellot Rubio, L. R., Balthasar, H., \& Collados, M. 2004, A\&A, 427, 319

Bellot Rubio, L. R., Schlichenmaier, R., \& Tritschler, A. 2006, A\&A, 453, 1117

Bello González, N. 2006, Ph.D. Thesis, University of Götingen

Bello González, N., \& Kneer, F. 2008, A\&A, 480, 265

Bello González, N., Flores Soriano, M., Kneer, F., \& Okunev, O. 2009, A\&A, 508, 941

Bello González, N., Kneer, F., \& Schlichenmaier, R. 2011, A\&A, in press, DOI: $10.1051 / 0004-6361 / 201118005$

Berger, T. E., \& Berdyugina, S. V. 2003, ApJ, 589, L117

Bernasconi, P. N., Rust, D. M., Georgoulis, M. K., \& Labonte, B. J. 2002, Sol. Phys., 209, 119

Bharti, L., Joshi, C., Jaaffrey, S. N. A., \& Jain, R. 2009, MNRAS, 393, 65

Bogdan, T. J., Gilman, P. A., Lerche, I., \& Howard, R. 1988, ApJ, 327, 451

Borrero, J. M., \& Kobel, P. 2011, A\&A, 527, A29

Brants, J. J., \& Steenbeek, J. C. M. 1985, Sol. Phys., 96, 229

Bray, R. J., \& Loughhead, R. E. 1964, Sunspots (Chapman Hall)

Cabrera Solana, D., Bellot Rubio, L. R., \& del Toro Iniesta, J. C. 2005, A\&A, 439,687

Cabrera Solana, D., Bellot Rubio, L. R., Beck, C., \& Del Toro Iniesta, J. C. 2007, A\&A, 475, 1067

Caligari, P., Moreno-Insertis, F., \& Schüssler, M. 1995, ApJ, 441, 886

Cheung, M. C. M., Schüssler, M., \& Moreno-Insertis, F. 2007, A\&A, 467, 703

Cheung, M. C. M., Schüssler, M., Tarbell, T. D., \& Title, A. M. 2008, ApJ, 687, 1373

Cheung, M. C. M., Rempel, M., Title, A. M., \& Schüssler, M. 2010, ApJ, 720, 233
Collados, M., Lagg, A., Díaz García, J. J., et al. 2007, in The Physics of Chromospheric Plasmas, ed. P. Heinzel, I. Dorotovič, \& R. J. Rutten, ASP Conf Ser., 368, 611

Danilovic, S., Schüssler, M., \& Solanki, S. K. 2010, A\&A, 509, A76

de Boer, C. R. 1996, A\&AS, 120, 195

del Toro Iniesta, J. C., Tarbell, T. D., \& Ruiz Cobo, B. 1994, ApJ, 436, 400

Elmore, D. F., Lites, B. W., Tomczyk, S., et al. 1992, in SPIE Conf. Ser., 1746, ed. D. H. Goldstein, \& R. A. Chipman, 22

Fang, F., Manchester, W., Abbett, W. P., \& van der Holst, B. 2010, ApJ, 714, 1649

Franz, M. 2011, Ph.D. Thesis, Albert-Ludwigs-University, Freiburg

Garcia de La Rosa, J. I. 1987, Sol. Phys., 112, 49

Giordano, S., Berrilli, F., Del Moro, D., \& Penza, V. 2008, A\&A, 489, 747

Gömöry, P., Beck, C., Balthasar, H., et al. 2010, A\&A, 511, A14

Grossmann-Doerth, U., Schüssler, M., \& Steiner, O. 1998, A\&A, 337, 928

Gurman, J. B., \& House, L. L. 1981, Sol. Phys., 71, 5

Hirzberger, J., Bonet, J. A., Sobotka, M., Vázquez, M., \& Hanslmeier, A. 2002, A\&A, 383, 275

Katsukawa, Y., Yokoyama, T., Berger, T. E., et al. 2007, PASJ, 59, 577

Keller, C. U., \& von der Lühe, O. 1992, A\&A, 261, 321

Keppens, R., \& Martinez Pillet, V. 1996, A\&A, 316, 229

Kosovichev, A. G. 2009, Space Sci. Rev., 144, 175

Kubo, M., Shimizu, T., \& Lites, B. W. 2003, ApJ, 595, 465

Kubo, M., Shimizu, T., \& Tsuneta, S. 2007, ApJ, 659, 812

Kucera, A., Beck, C., Gömöry, P., et al. 2008, in 12th European Solar Physics Meeting, Freiburg, Germany, 2

Kurucz, R. L., Furenlid, I., Brault, J., \& Testerman, L. 1984, Solar Flux Atlas From $296 \mathrm{~nm}$ to $1300 \mathrm{~nm}$ (National Solar Observatory)

Leka, K. D. 1997, ApJ, 484, 900

Leka, K. D., \& Skumanich, A. 1998, ApJ, 507, 454

Lites, B. W. 2009, Space Sci. Rev., 144, 197

Lites, B. W., \& Skumanich, A. 1990, ApJ, 348, 747

Lites, B. W., Elmore, D. F., Seagraves, P., \& Skumanich, A. P. 1993, ApJ, 418, 928

Lites, B. W., Low, B. C., Martinez Pillet, V., et al. 1995, ApJ, 446, 877

Lites, B. W., Scharmer, G. B., Berger, T. E., \& Title, A. M. 2004, Sol. Phys., 221,65

Lites, B. W., Kubo, M., Socas-Navarro, H., et al. 2008, ApJ, 672, 1237

Louis, R. E., Bayanna, A. R., Mathew, S. K., \& Venkatakrishnan, P. 2008, Sol. Phys., 252, 43

Louis, R. E., Bellot Rubio, L. R., Mathew, S. K., \& Venkatakrishnan, P. 2009, ApJ, 704, L29

Martínez-Sykora, J., Hansteen, V., \& Carlsson, M. 2009, ApJ, 702, 129

Mathew, S. K., Lagg, A., Solanki, S. K., et al. 2003, A\&A, 410, 695

Metcalf, T. R. 1994, Sol. Phys., 155, 235

Metcalf, T. R., Leka, K. D., Barnes, G., et al. 2006, Sol. Phys., 237, 267

Nagata, S., Tsuneta, S., Suematsu, Y., et al. 2008, ApJ, 677, L145

Nave, G., Johansson, S., Learner, R. C. M., Thorne, A. P., \& Brault, J. W. 1994, ApJS, 94, 221

Ortiz, A., Bellot Rubio, L. R., \& Rouppe van der Voort, L. 2010, ApJ, 713, 1282

Otsuji, K., Shibata, K., Kitai, R., et al. 2007, PASJ, 59, 649

Paletou, F., Lafon, M., Maeght, P., et al. 2007 [arXiv:0712.2421]

Pariat, E., Aulanier, G., Schmieder, B., et al. 2004, ApJ, 614, 1099

Parker, E. N. 1955, ApJ, 121, 491

Parker, E. N. 1979, Cosmical Magnetic Fields: Their Origin and Thier Activity (NewYork: Oxford University Press)

Puschmann, K. G., \& Sailer, M. 2006, A\&A, 454, 1011

Puschmann, K. G., Kneer, F., Seelemann, T., \& Wittmann, A. D. 2006, A\&A, 451,1151

Puschmann, K. G., Ruiz Cobo, B., \& Martínez Pillet, V. 2010, ApJ, 720, 1417

Rezaei, R. 2008, Ph.D. Thesis, Albert-Ludwigs University, Freiburg

Rezaei, R., Schlichenmaier, R., Beck, C., \& Bellot Rubio, L. R. 2006, A\&A, 454, 975

Rezaei, R., Schlichenmaier, R., Beck, C., Bruls, J., \& Schmidt, W. 2007, A\&A, 466, 1131

Rimmele, T. R. 1997, ApJ, 490, 458

Rouppe van der Voort, L., Bellot Rubio, L. R., \& Ortiz, A. 2010, ApJ, 718, L78

Rucklidge, A. M., Schmidt, H. U., \& Weiss, N. O. 1995, MNRAS, 273, 491

Rüedi, I., Solanki, S. K., \& Livingston, W. 1995, A\&A, 302, 543

Ruiz Cobo, B., \& del Toro Iniesta, J. C. 1992, ApJ, 398, 375

Rutten, R. J., Hammerschlag, R. H., Bettonvil, F. C. M., Sütterlin, P., \& de Wijn, A. G. 2004, A\&A, 413, 1183

Sánchez Cuberes, M., Puschmann, K. G., \& Wiehr, E. 2005, A\&A, 440, 345

Scharmer, G. B. 2009, Space Sci. Rev., 144, 229

Scherrer, P. H., Bogart, R. S., Bush, R. I., et al. 1995, Sol. Phys., 162, 129

Schlichenmaier, R. 1997, Ph.D. Thesis, Ludwig-Maximilians-Univ. München

Schlichenmaier, R. 2009, Space Sci. Rev., 144, 213

Schlichenmaier, R., \& Collados, M. 2002, A\&A, 381, 668 
Schlichenmaier, R., Bello González, N., Rezaei, R., \& Waldmann, T. A. 2010a, AN, 331, 563

Schlichenmaier, R., Rezaei, R., Bello González, N., \& Waldmann, T. A. 2010b, A\&A, 512, L1

Schlichenmaier, R., Bello González, N., \& Rezaei, R. 2011, in Physics of Sun and Starspots, ed. D. P. Choudhary, \& K. G. Strassmeier, IAU Symp., 273, 134

Schmidt, H. U. 1991, Geophys. Astrophys. Fluid Dyn., 62, 249

Schröter, E. H., Soltau, D., \& Wiehr, E. 1985, Vistas Astron., 28, 519

Schüssler, M. 1984, A\&A, 140, 453

Schüssler, M., \& Vögler, A. 2006, ApJ, 641, L73

Shimizu, T., Katsukawa, Y., Kubo, M., et al. 2009, ApJ, 696, L66

Sobotka, M., Bonet, J. A., \& Vazquez, M. 1993, ApJ, 415, 832

Sobotka, M., Bonet, J. A., \& Vazquez, M. 1994, ApJ, 426, 404

Sobotka, M., Brandt, P. N., \& Simon, G. W. 1997, A\&A, 328, 682

Solanki, S. K. 2003, A\&ARv, 11, 153

Solanki, S. K., \& Schmidt, H. U. 1993, A\&A, 267, 287

Spruit, H. C. 1979, Sol. Phys., 61, 363

Strous, L. H., \& Zwaan, C. 1999, ApJ, 527, 435

Tomczyk, S., Elmore, D. F., Lites, B. W., et al. 1992, BAAS, 24, 814

Tortosa-Andreu, A., \& Moreno-Insertis, F. 2009, A\&A, 507, 949
Tritschler, A. 2009, in ASP Conf. Ser., ed. B. Lites, M. Cheung, T. Magara, J. Mariska, \& K. Reeves, 415, 339

von der Lühe, O., Soltau, D., Berkefeld, T., \& Schelenz, T. 2003, in Innovative Telescopes and Instrumentation for Solar Astrophysics. ed. S. L. Keil, \& S. V. Avakyan, SPIE, 4853, 187

Wang, H., \& Zirin, H. 1992, Sol. Phys., 140, 41

Weiss, N. O. 1966, Roy. Soc. London Proc. Series A, 293, 310

Weiss, N. O. 2006, Space Sci. Rev., 124, 13

Weiss, N. O., Thomas, J. H., Brummell, N. H., \& Tobias, S. M. 2004, ApJ, 600, 1073

Wentzel, D. G. 1992, ApJ, 388, 211

Westendorp Plaza, C., del Toro Iniesta, J. C., Ruiz Cobo, B., et al. 2001, ApJ, 547,1130

Wöger, F., \& von der Lühe, O. 2008, in SPIE Conf. Ser., 7019

Wöger, F., von der Lühe, O., \& Reardon, K. 2008, A\&A, 488, 375

Yang, G., Xu, Y., Wang, H., \& Denker, C. 2003, ApJ, 597, 1190

Zwaan, C. 1985, Sol. Phys., 100, 397

Zwaan, C. 1987, ARA\&A, 25, 83

Zwaan, C. 1992, in Sunspots. Theory and Observations, ed. J. H. Thomas, \& N. O. Weiss, NATO ASIC Proc. 375, 75 